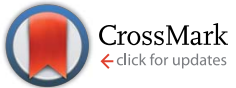

Cite this: RSC Adv., 2015, 5, 61787
Received 28th March 2015 Accepted 10th July 2015

DOI: $10.1039 / c 5 r a 05534 b$

www.rsc.org/advances

\section{Phycocyanobilin, a bioactive tetrapyrrolic compound of blue-green alga Spirulina, binds with high affinity and competes with bilirubin for binding on human serum albumin $\uparrow$}

\author{
Simeon L. Minic, $\mathfrak{t}^{\mathrm{a}}$ Miloš Milcic, $\mathfrak{t}^{\mathrm{a}}$ Dragana Stanic-Vucinic, ${ }^{a}$ Milica Radibratovic, ${ }^{b}$ \\ Theodore G. Sotiroudis, ${ }^{c}$ Milan R. Nikolic ${ }^{* a}$ and Tanja Ćirković Velickovic ${ }^{a}$
}

Human serum albumin (HSA) is an important regulator of the pharmacokinetic properties of bioactive compounds. Phycocyanobilin is a blue tetrapyrrole chromophore of C-phycocyanin with proven healthpromoting activities. Despite its structural similarity to bilirubin, the conformation it adopts in aqueous solution is different and the pigment is more soluble than bilirubin. The aim of our study was to examine binding of phycocyanobilin for HSA and to investigate its competition with bilirubin. Based on a computational approach, we demonstrated two putative high-affinity binding pockets on HSA of virtually identical energies for the neutral and anion forms of bilirubin, but with slightly favorable predictions for anion forms of phycocyanobilin. Computational prediction of phycocyanobilin $\mathrm{p} K_{\mathrm{a}}$ values suggested a monoanion form to be the most stable form at physiological conditions. The computationally predicted binding sites for phycocyanobilin were identical to the two previously identified binding sites for bilirubin (subdomains IB and IIA). Results obtained by protein and pigment fluorescence measurements, circular dichroism, and competition experiments confirmed high affinity (binding constant of $2.2 \times 10^{6} \mathrm{M}^{-1}$ at $25{ }^{\circ} \mathrm{C}$ ), stereoselective binding of phycocyanobilin $M$-conformer to HSA and its competition with bilirubin, warfarin and hemin. Our experimental data confirm that phycocyanobilin binds to IB and IIA binding site of HSA with an affinity similar to bilirubin. In conditions characterized by an increased bilirubin plasma concentration, or intake of drugs binding to IB or IIA binding site, pharmacokinetics of phycocyanobilin may also be changed.

\section{Introduction}

The intensive blue color of photosynthetic cyanobacteria Spirulina arises from C-phycocyanin, the most abundant protein of this blue-green alga with exceptional nutritional properties. ${ }^{1}$ It has been documented that the C-phycocyanin's healthbeneficial activities are exerted by its chromophore, phycocyanobilin. ${ }^{2,3}$ The chemical structure of phycocyanobilin is highly similar to that of bile pigment bilirubin and its precursor, biliverdin (Fig. 1A).

Extraordinary binding capacity of human serum albumin (HSA) for various ligands makes this protein an important

\footnotetext{
${ }^{a}$ Department of Biochemistry, Center of Excellence for Molecular Food Sciences, Faculty of Chemistry, University of Belgrade, Studentski trg 12-16, 11000 Belgrade, Serbia. E-mail: mnikolic@chem.bg.ac.rs; Fax: +381 11 2184330; Tel: +381 113336657 ${ }^{b}$ Institute of Chemistry, Technology and Metallurgy - Center for Chemistry, University of Belgrade, Njegoševa 12, 11000 Belgrade, Serbia

'Institute of Biology, Medicinal Chemistry and Biotechnology, National Hellenic Research Foundation, 48 Vassileos Constantinou Ave., 11635 Athens, Greece

$\dagger$ Electronic supplementary information (ESI) available. See DOI: 10.1039/c5ra05534b

\$ These two authors contributed equally to this work.
}

regulator of the pharmacokinetic properties of drugs and a model protein to study interactions with natural bioactive compounds. HSA is a single polypeptide globular protein composed of three homologous $\alpha$-helical domains (I, II and III), each containing two subdomains (A and B). Binding sites for most aromatic and heterocyclic ligands are mainly located within two hydrophobic pockets in subdomains IIA (Sudlow's site I) and IIIA (Sudlow's site II). ${ }^{4,5}$ Sudlow's site I is known as the warfarin-azapropazone site. ${ }^{4}$ The characteristic feature of this site is binding of the bulky heterocyclic anion ligand with a negative charge localized in the middle of the molecule. Drugs binding in this site include warfarin, valproate and azapropazone. Site II is known as the indole-benzodiazepine site, and ligands that bind to this region include ibuprofen, flurbiprofen and diazepam. ${ }^{4}$ There are also drugs that bind to both sites on HSA. ${ }^{4,6}$

The biosynthetic form of the bilirubin molecule (4Z,15Z-bilirubin IX $\alpha$ ), which is lipophilic and insoluble in water at neutral $\mathrm{pH}$, is transported in blood plasma by serum albumin to the liver where it is converted to a water-soluble glucuronide. ${ }^{7}$ Bilirubin is normally present in plasma at low concentration $(5-17 \mu \mathrm{M})$ but, when conjugation of bilirubin is 


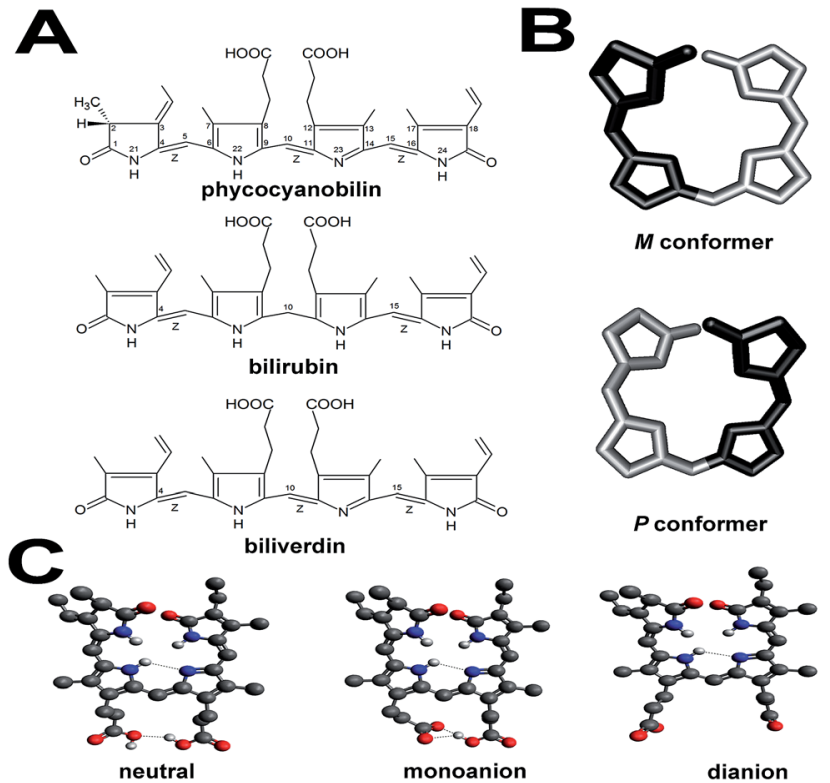

Fig. 1 (A) Chemical structure of phycocyanobilin, bilirubin and biliverdin; (B) schematic representation of opposite helical conformations ( $M$ or $P$ ) of phycocyanobilin: half of the molecules drawn by bold are above the plane of the paper. Ring substituents were omitted for clarity; (C) conformations of differently ionized (neutral, monoanionic and dianionic) forms of phycocyanobilin.

deficient, the unconjugated pigment accumulates in the circulation and extravascular tissue. HSA acts as a physiological buffer for bilirubin as it prevents the transfer of bilirubin from blood to tissues and impairs the development of bilirubin encephalopathy, a serious pathological condition. ${ }^{8}$ Evidence from circular dichroism (CD) studies shows that binding of bilirubin to HSA is enantioselective, since the $P$-enantiomer is bound preferentially. ${ }^{9}$ However, biliverdin IX $\alpha$, a biological precursor of bilirubin, binds to HSA preferentially as $M$-enantiomer. ${ }^{10}$

The biosynthetic $4 Z, 15 Z$-isomer of bilirubin binds with high affinity (the binding constant of $10^{7}$ to $10^{8} \mathrm{M}^{-1}$ ) to a single site on HSA. ${ }^{11,12}$ There are also one or two secondary sites with affinities that are at least tenfold lower. ${ }^{\mathbf{8} 13}$ This issue is complicated by the photoisomerization of bilirubin, a process that is relevant to the clinical management of metabolic disorders involving the pigment. The conversion of $4 Z, 15 Z$-bilirubin to $4 Z, 15 E$-bilirubin-IX $\alpha$ ( $4 Z, 15 E$-bilirubin) by photosensitization results in the formation of a geometric isomer that has a lower binding affinity for HSA but higher water solubility, which permits it to be excreted more easily than the precursor 4Z,15Z-bilirubin. ${ }^{14}$

Despite numerous studies, there is controversy about the localization of the primary high-affinity bilirubin-binding site on albumin. A crystallographic study located binding site for bilirubin photoisomer $4 Z, 15 E$-bilirubin within the subdomain IB. This modeled conformation has $M$-type helicity, which is opposite to the $P$-type helicity observed in CD measurements of $4 Z, 15 Z$-bilirubin bound to HSA. ${ }^{15}$ In line with this, numerous studies pointed to the hydrophobic pocket of the subdomain IIA as the primary high-affinity binding site for bilirubin. ${ }^{8,16} \mathrm{Hemin}$, another precursor of bilirubin, predominantly binds to site IB on HSA. ${ }^{17,18}$

Another controversy about bilirubin is its charge state at the physiological $\mathrm{pH}$. The most recent studies demonstrated that despite the experimental data that supported lower $\mathrm{p} K_{\mathrm{a}}$ values of the two carboxylic groups in the molecule, the charge state of bilirubin is neutral until $\mathrm{pH} 8$, i.e. at physiological $\mathrm{pH}$ of 7.2-7.4. ${ }^{19}$ The molecule exists in a tight hydrogen-bonded conformation that prevents ionization of its carboxyl groups and reduces solubility in aqueous solutions. No similar data exist for bilirubin-analog phycocyanobilin. Similarly to biliverdin, phycocyanobilin cannot adopt ridge-tile conformation of bilirubin because of the presence of the double bond that prevents rotation and also give rise to quite different spectral characteristics of the intensive blue colored pigment. ${ }^{20}$

Our work, based on a computational approach for studying interactions between HSA, bilirubin and bilirubin-analog phycocyanobilin, demonstrated two putative high-affinity binding pockets on HSA for bilirubin and phycocyanobilin, that could accommodate both neutral and anion forms of both molecules. The binding sites were identical to the two previously identified binding sites for bilirubin (sites IB and IIA). Results obtained by protein and pigment fluorescence measurements, circular dichroism, as well as bilirubindisplacement experiments, confirmed high affinity, enantioselective binding of phycocyanobilin $M$-conformer to HSA and its competition with bilirubin, warfarin and hemin for the binding on HSA.

\section{Materials and methods}

\section{Materials}

HSA (purity $\geq 97 \%$ ), bilirubin (purity $\geq 98 \%$ ), warfarin (purity $\geq 98 \%$ ) and hemin (purity $\geq 98 \%$ ) were purchased from SigmaAldrich (Taufkirchen, Germany) and used without further purification. HSA solutions were prepared in $20 \mathrm{mM}$ Tris buffer, $\mathrm{pH} 7.4$ and the protein concentration was determined using an extinction coefficient of $35700 \mathrm{M}^{-1} \mathrm{~cm}^{-1}$ at $280 \mathrm{~nm}$. Bilirubin, warfarin and hemin were dissolved in $99.9 \%$ dimethyl sulfoxide (DMSO) to make $4 \mathrm{mM}$ stock solution. Phycocyanobilin was isolated and purified from commercial Hawaiian Spirulina Pacifica powder (Nutrex, USA) and characterized as previously described. ${ }^{21}$ Stock phycocyanobilin solutions were obtained by dissolving dry pigment in anhydrous methanol. Phycocyanobilin concentration was determined by measuring absorbance of aliquots diluted in conc. $\mathrm{HCl} / \mathrm{MeOH}(1: 19 ; \mathrm{v} / \mathrm{v})$ at $680 \mathrm{~nm}$ $\left(\varepsilon=37900 \mathrm{M}^{-1} \mathrm{~cm}^{-1}\right) \cdot{ }^{22}$ For all experiments, stock solutions of HSA, phycocyanobilin, bilirubin, warfarin and hemin were diluted with $20 \mathrm{mM}$ Tris buffer, $\mathrm{pH} 7.4$ and final concentrations of methanol and DMSO in the protein-ligand mixtures did not exceed $1 \%(\mathrm{v} / \mathrm{v})$. Phycocyanobilin, bilirubin and hemin stock solutions and mixtures were constantly kept in the dark. All other chemicals were of analytical reagent grade and Milli-Q water (Millipore, Molsheim, France) was used throughout the experiments. 


\section{Computational details}

$\mathrm{p} K_{\mathrm{a}}$ values of phycocyanobilin were estimated using isodesmic reaction approach, ${ }^{23}$ i.e. calculation of free energy of proton exchange reaction in water solution (the reaction schemes (I) and (II)) with bilirubin as reference specie.

$$
\begin{aligned}
& \mathrm{PCBH}_{2(\text { soln })}+\mathrm{BRH}_{(\text {soln })}{ }^{-} \rightarrow \mathrm{PCBH}_{(\text {soln })}{ }^{-}+\mathrm{BRH}_{2(\text { soln })} \\
& \mathrm{PCBH}_{(\text {soln })}{ }^{-}+\mathrm{BR}_{(\text {soln })}{ }^{2-} \rightarrow \mathrm{PCB}_{(\text {soln })}{ }^{2-}+\mathrm{BRH}_{(\text {soln })}{ }^{-}
\end{aligned}
$$

The structures of neutral phycocyanobilin (in most stable helical all-Z, all-syn conformation) and bilirubin (in the biosynthetic $4 Z, 15 Z$ "ridge-tile" conformation) and corresponding anions were fully optimized with B3LYP densityfunctional theory method, using standard 6-311++G(d,p) basis set and characterized as energy minima by the absence of imaginary frequencies. The solvent effects have been taken into account in all geometry optimizations and energy calculations by using the SMD model. ${ }^{24}$ All quantum chemical calculations were performed with Gaussian09 program package.

Molecular modeling of the phycocyanobilin/bilirubin-HSA complex was performed using the 3D crystal structure of HSA (PDB ID: 1BM0) extracted from the Protein Data Bank. ${ }^{25}$ All water molecules were removed from the protein structure. Since the hydrogen data is missing, protonation state of each titratable amino acid was estimated with finite difference PoissonBoltzmann continuum electrostatics model as implemented in $\mathrm{H}++3.0$ program. ${ }^{26}$ Missing amino acids from $\mathrm{N}$ - and C-terminus were built and the whole protein structure was optimized in CHARMM program (version c35b1) using the CHARM22 protein force field. ${ }^{27}$ Optimization of HSA structure was carried out in 2000 optimization steps (1000 steps with steepest-descent algorithm followed by 1000 steps with Newton-Raphson algorithm).

The optimized structures of neutral ligands and corresponding anions were taken from $\mathrm{p} K_{\mathrm{a}}$ calculations described earlier. Optimized structures of protein and ligands were further subjected to AutoDockTools (version 1.5.6. Sep_17_14) program for docking preparation. All protein residues were kept rigid. For phycocyanobilin ligand all three double bond were kept rigid in $Z$ conformation, while the three single bonds of the exocyclic methine bridges were set to be rotational. Similarly, bilirubin double bonds 4 and 15 (Fig. 1A) were kept in $Z$ conformation and all single bonds were set to be rotational. A grid box, with the dimensions $28 \times 28 \times 28 \AA$, was used to accommodate the ligand to move freely during docking run. In order to cover whole surface and volume of HSA a grid box was moved over the rectangular matrix containing protein with points $8 \AA$ apart, so the total of 1188 docking runs were produced for every ligand. The docking studies were carried out with AutoDockVina program (version 1.1.2). ${ }^{28}$ To increase the probability for finding the minimum during docking run exhaustiveness parameter in AutoDock Vina program was set to 100. From each docking run 9 binding modes with highest scoring function were kept for further analysis.

Analysis of data from PDB recognized a total of 7 fatty acid binding sites (FA1-FA7), 6 other ligands binding sites (IB, IIA,
IIA-IIB, IIIA, IIIB and cleft) and two drug binding sites (drug site 1 and drug site 2). ${ }^{29}$ Based on crystallographic data, ligand type and ligand binding site(s), a subset of 16 HSA crystal structures of total of 96 structures found in PDB was chosen for further data analysis (Table S1 of ESI $\dagger$ ). Overlapping amino acid residues that can be included in binding both phycocyanobilin and other ligands were collected (Table S2 of ESI $\dagger$ ). Amino acid residues from binding sites IB and IIA and selected ligands are presented in the Fig. $\mathrm{S} 1$ (in ESI $\dagger$ ).

In order to verify validity of used molecular modeling method, an extensive docking study on selected HSA crystal structures was conducted. The docking method was the same as described above, with two changes: (i) the structure of the protein was not optimized and (ii) in order to save the computational time, the exhaustiveness parameter in AutoDockVina program was set to 10 . To verify the accuracy of the method, a re-docking simulation was performed for every ligand found in the selected crystal structures, except for myristic acid. Obtained data have shown that AutoDockVina program is performing very well in finding the proper binding site for studied ligands. RMSD values between center of the mass of docked and crystalographically found ligand positions are presented in Table S3 (in ESI $\dagger$ ).

For the 19 out of 21 ligands found in selected crystal structures in re-docked analyses AutoDockVina was able to find the proper position and orientation of the ligand and RMSD values are lower than $1 \AA$ (Table S3 and Fig. S2A of ESI $\dagger$ ). For the two remaining ligands (site IIIB in 2 VUF and site IB in 4LA0) a proper position for the ligand was found but with different orientation (Fig. S2B of ESI $\dagger$ ), resulting in a somewhat larger RMSD values (Table S3 of ESI $\dagger$ ).

The influence of conformational changes in protein due to ligand binding on HSA affinity to bind phycocyanobilin was examined. In this simulation, all crystalographically found ligands and fatty acids coordinates were deleted from crystal structures and docking studies were conducted on ligand-free protein using monoanionic form of phycocyanobilin.

To examine an influence of bound fatty acids or other ligands on phycocyanobilin binding to HSA, final docking study on 6 selected crystal structures with myristic acid and 14 structures with bound other ligands was done.

\section{Fluorescence spectroscopy measurements}

All fluorescence data were obtained on FluoroMax®-4 spectrofluorometer (HORIBA Scientific, Japan) under thermostated conditions, with the width of the excitation and emission slit both adjusted at $5 \mathrm{~nm}$.

Binding of phycocyanobilin to HSA was studied by the fluorescence quenching titration method using the intrinsic fluorescence of HSA as a probe, at constant protein $(0.375 \mu \mathrm{M})$ and various ligand concentrations (0 to $1.5 \mu \mathrm{M})$. The steady-state fluorescence spectra were measured at 25,30 and $37{ }^{\circ} \mathrm{C}$. The excitation wavelength was set at $280 \mathrm{~nm}$ (excitation of the Trp and Tyr), and the emission spectra were read at 290 to $400 \mathrm{~nm}$. The appropriate blanks corresponding to the various phycocyanobilin in buffer concentration were subtracted to correct 
background of fluorescence. Fluorescence intensities were corrected for the absorption of excited light and the re-absorption of emitted light due to the inner-filter effect, according to relation: ${ }^{30}$

$$
F_{\mathrm{c}}=F_{0} 10^{\left(A_{\mathrm{em}}+A_{\mathrm{ex}}\right) / 2}
$$

where $F_{0}$ is measured fluorescence, $F_{\mathrm{c}}$ is corrected fluorescence, $A_{\text {ex }}$ and $A_{\text {em }}$ are absorbance of quencher at excitation and pick emission wavelength $(340 \mathrm{~nm})$, respectively.

To determine the type of quenching, Stern-Volmer's (SV) constants were calculated at different temperatures using the equation: ${ }^{30}$

$$
\frac{F_{0}}{F}=1+k_{\mathrm{q}} \tau_{\mathrm{o}}[\mathrm{Q}]=1+K_{\mathrm{SV}}[\mathrm{Q}]
$$

where $F_{0}$ and $F$ are protein emission fluorescence at $340 \mathrm{~nm}$ without and with addition of ligand, respectively, $k_{\mathrm{q}}$ is quenching rate constant of the biomolecule, $\tau_{\mathrm{o}}$ is average lifetime of the biomolecule without quencher $\left(10^{-8} \mathrm{~s}\right),[\mathrm{Q}]$ is total quencher (phycocyanobilin) concentration, and $K_{\mathrm{SV}}$ is $\mathrm{SV}$ quenching constant. $K_{\mathrm{SV}}$ can be calculated on the basis of SV plots.

The estimation of the association (binding) constant $\left(K_{\mathrm{a}}\right)$, and number of binding sites ( $n$ ) of HSA-phycocyanobilin system was done using equation: ${ }^{31}$

$$
\log \frac{F_{0}-F}{F}=-n \log \frac{1}{[\mathrm{~L}]-[\mathrm{P}] \frac{F_{0}-F}{F_{0}}}+n \log K_{\mathrm{a}}
$$

where $[\mathrm{P}]$ and $[\mathrm{L}]$ are total concentration of protein (HSA) and ligand (phycocyanobilin), respectively, and $K_{\mathrm{a}}$ is the binding constant.

The synchronous fluorescence characteristics of HSA-phycocyanobilin complex were recorded at two different scanning intervals: $\Delta \lambda 15 \mathrm{~nm}$ (Tyr residues excitation) and $\Delta \lambda 60 \mathrm{~nm}(\operatorname{Trp}$ residue excitation), where $\Delta \lambda=\lambda_{\mathrm{EM}}-\lambda_{\mathrm{EX}}$.

The thermodynamic parameters were determined from the equations:

$$
\begin{aligned}
& \ln K_{\mathrm{a}}=-\frac{\Delta H}{R T}+\frac{\Delta S}{R} \\
& \Delta G=\Delta H-T \Delta S
\end{aligned}
$$

where $K_{\mathrm{a}}$ is the binding constant, $R$ is universal gas constant (8.313 $\mathrm{J} \mathrm{mol}^{-1} \mathrm{~K}^{-1}$ ), $\Delta G$ is free energy change, $\Delta H$ is enthalpy change, and $\Delta S$ is entropy change. The values of $\Delta H$ and $\Delta S$ were calculated from the slope and intercept of linear plot of $\ln K_{\mathrm{a}}$ versus $1 / T$.

Enhancing of phycocyanobilin $(1 \mu \mathrm{M})$ emission fluorescence by HSA (up to $2 \mu \mathrm{M}$ ) was recorded in the range of 470 to $650 \mathrm{~nm}$, where excitation wavelength was set to $460 \mathrm{~nm}$. Affinity constant for ligand binding to protein was calculated from enhancement of phycocyanobilin fluorescence at $547 \mathrm{~nm}$, using the equation: ${ }^{32}$

$$
\frac{1}{\Delta F}=\frac{1}{\Delta F_{\max }}+\frac{1}{K_{\mathrm{a}} \Delta F_{\max }[\mathrm{P}]}
$$

where $\Delta F$ is the change of phycocyanobilin fluorescence intensity in the presence and absence of HSA; $\Delta F_{\max }$ is the maximal change of fluorescence intensity, $K_{\mathrm{a}}$ is the binding constant, and $[\mathrm{P}]$ is the concentration of HSA.

Bilirubin displacement from HSA-bilirubin complex by phycocyanobilin was investigated by recording synchronous fluorescence spectra in the range $490-650 \mathrm{~nm}$, where $\Delta \lambda$ was 56 $\mathrm{nm}$. Equimolar mixture of HSA and bilirubin $(4 \mu \mathrm{M})$ was titrated with phycocyanobilin in the range of phycocyanobilin to HSA-bilirubin complex molar ratio 0-2.5. Phycocyanobilin displacement from HSA-phycocyanobilin complex by bilirubin was also investigated by recording synchronous fluorescence spectra in the range $590-750 \mathrm{~nm}$, where $\Delta \lambda$ was $87 \mathrm{~nm}$. Equimolar mixture of HSA and phycocyanobilin $(2 \mu \mathrm{M})$ was titrated with bilirubin in the range of bilirubin to HSA-phycocyanobilin complex molar ratio 0-2.5.

In order to confirm that phycocyanobilin and bilirubin bind to warfarin-azapropazone binding site on HSA, equimolar mixtures of HSA and phycocyanobilin or HSA and bilirubin $(2 \mu \mathrm{M})$ were titrated with warfarin in the range of warfarin to HSA-phycocyanobilin/bilirubin complex molar ratio 0-10. Fluorescence spectra were recorded in the range $480-585 \mathrm{~nm}$, whereas the excitation wavelength was $460 \mathrm{~nm}$. Also, equimolar mixture of HSA and warfarin $(0.25 \mu \mathrm{M})$ was titrated with phycocyanobilin or bilirubin up to ligand-complex molar ratio 2 , and synchronous fluorescence spectra were obtained in the range $365-400 \mathrm{~nm}$, where $\Delta \lambda$ was $64 \mathrm{~nm}$. Finally, to confirm that phycocyanobilin and bilirubin bind to IB binding site on HSA, equimolar mixtures of HSA and phycocyanobilin or HSA and bilirubin $(2 \mu \mathrm{M})$ were titrated with hemin in the range of hemin to HSA-phycocyanobilin/bilirubin complex molar ratio 0-2.5. Fluorescence spectra were recorded in the range $480-585 \mathrm{~nm}$, whereas the excitation wavelength was $460 \mathrm{~nm}$.

For the determination of binding sites, titration of phycocyanobilin to HSA in absence and presence of site markers (warfarin and hemin) were performed as a competitive experiment. 0.5 $\mu \mathrm{M}$ HSA was pre-incubated with $0.5 \mu \mathrm{M}$ site markers and phycocyanobilin was gradually added to the HSA-site markers up to four-fold molar excess. Phycocyanobilin binding constants were calculated using eqn (3) as described above.

All experiments were replicated at least twice, with duplicate repeated measures within each replication.

\section{CD spectroscopy measurements}

The CD measurements were carried out on Jasco J-815 spectropolarimeter (Jasco, Tokyo, Japan). For the measurements in the near-UV and visible region (260-750 nm), with two scans averaged for each CD spectra, concentrations of HSA and phycocyanobilin were $18 \mu \mathrm{M}$, and for displacement experiments bilirubin concentrations were $18 \mu \mathrm{M}$ and $36 \mu \mathrm{M}$.

\section{Results and discussion}

\section{Calculation of phycocyanobilin $\mathrm{p}_{\mathrm{a}}$ values}

Chemical structures of intensively blue-colored phycocyanobilin, blue-green biliverdin and orange-yellow bile pigment 
bilirubin are highly similar (Fig. 1A). Moreover, $P$ - and $M$-helical enantiomers of phycocyanobilin are presented in Fig. 1B.

There is a lot of controversy in the literature about ionization state of two bilirubin carboxylic groups at physiological conditions. ${ }^{19}$ No similar experimental or theoretical data exist for phycocyanobilin, but based on structural similarity and proximity of carboxylate groups in biliverdin, a structural analog of phycocyanobilin, it is more likely that $\mathrm{p} K_{\mathrm{a}}$ values of phycocyanobilin will not be similarly high.

Our data confirmed high $\mathrm{p} K_{\mathrm{a}}$ values $\left(\mathrm{p} K_{\mathrm{a} 1}=8.1\right.$ and $\left.\mathrm{p} K_{\mathrm{a} 2}=8.4\right)$ recently predicted for bilirubin. ${ }^{19}$ It is evident from the published data and our computational predictions that ridge-tile conformation adopted by bilirubin in aqueous solutions presents an obstacle to ionization of its carboxylic groups. Contrary to bilirubin, phycocyanobilin's most stable conformation is helicoidal and enables easy ionization of the first carboxylic group of the pigment. $\mathrm{p} K_{\mathrm{a} 1}$ value predicted by quantum chemical calculations using isodesmic reaction approach for phycocyanobilin is 2.4. Formation of the hydrogen bond between ionized and protonated group (Fig. 1C) retracts ionization of the second carboxylic group and the estimated $\mathrm{p} K_{\mathrm{a} 2}$ is 9.6. Thus, the most compact and the most stable conformation of phycocyanobilin in aqueous solutions and at physiological pH is its monoanion form, in which one hydrogen atom is bridging two carboxylate groups (Fig. 1C).

\section{Docking analysis of high-affinity binding sites for phycocyanobilin and bilirubin on HSA}

Six different docking studies were performed, either with fully protonated phycocyanobilin and bilirubin molecules or with their mono and dianionic forms. Analysis of the docking results predicted two potent binding sites (subdomains IB and IIA) for phycocyanobilin on HSA molecule (Fig. 2A), and they overlap with those previously described of bilirubin (Fig. 2B). Estimated binding energies are slightly higher for bilirubin binding comparing to phycocyanobilin and for both anionic pigment forms than for the neutral molecules. It can be observed that virtually identical energies were estimated for the neutral and anion forms of bilirubin, but with slightly favorable predictions for anion forms of phycocyanobilin (Table 1).

It is well known that binding of various ligands induce conformational changes in HSA. Therefore, we have examined the influence of different HSA conformations on phycocyanobilin binding, using 16 HSA crystal structures. Two proposed phycocyanobilin binding sites (IB and IIA) are most probable binding sites regardless of the protein conformation, although the estimated binding energies are somewhat influenced by protein conformation and positions of amino acids side chains; for IB site binding energies are between 8.4 and $10.8 \mathrm{kcal} \mathrm{mol}^{-1}$ and for IIA between 8.5 and $10.5 \mathrm{kcal} \mathrm{mol}^{-1}$ (Table S3 of ESI $\dagger$ ).

The first high-affinity binding site for phycocyanobilin is located at the IB subdomain of the protein and is composed of the residues from helices $\mathrm{H} 8, \mathrm{H} 9, \mathrm{H} 10$ and polypeptide "strap" (residues 110-117) connecting subdomains IB and IA. The binding site is in L-shaped geometry, and phycocyanobilin ligand structure with highest binding energy adopts $M$ conformation (Fig. 2C). Interior of the binding site is mainly
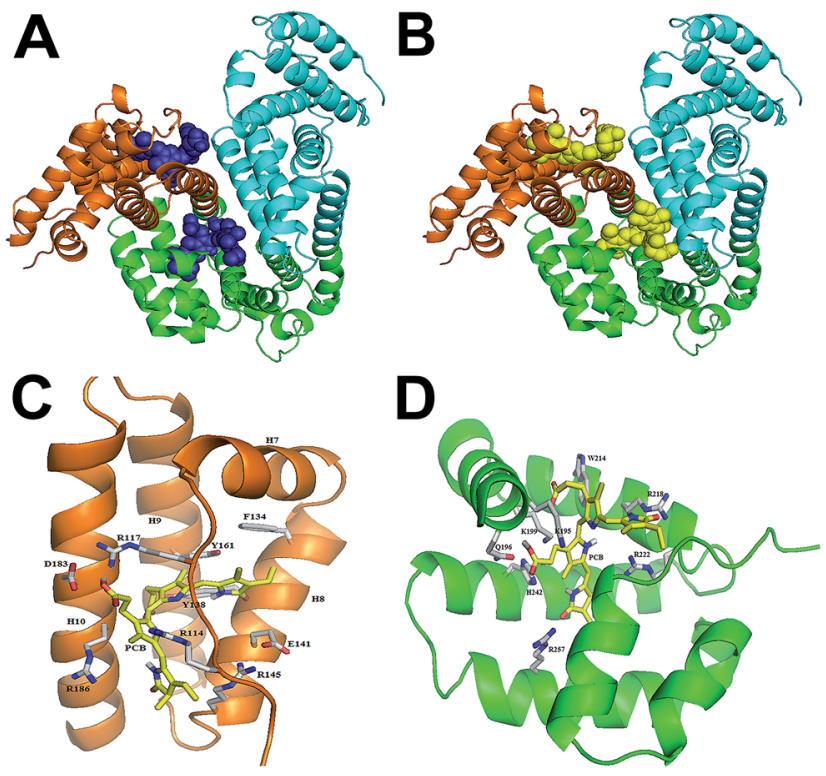

Fig. 2 Molecular modeling of phycocyanobilin/bilirubin bound human HSA. The domains in ribbon model of protein HSA (PDB ID: 1BMO) are color codes as follows: I, orange; II, green; III, turquoise blue. Structural models depicting two potential binding sites for monoanionic form of phycocyanobilin (A) and bilirubin (B) to HSA: bound phycocyanobilin and bilirubin are shown in purple and yellow color space filling representation, respectively. The best docked results for phycocyanobilin (represented using a stick model) complexed to HSA: subdomain IB (C) and subdomain IIA (D). The secondary structure of the protein is shown, and important neighboring amino acids residues within $3.5 \AA$ are labeled. Hydrogen atoms were omitted for clarity. The figures have been generated using PyMOL (http://www.pymol.org/).

hydrophobic with main contribution from Leu-115, Pro-118, Met-123, Ile-142, Phe-157 and Leu-182. There are three positively charged amino acids at the entrance of the binding site: Arg-114, Arg-117 and Arg-186. Our docking study indicates Arg-114 and Arg-117 are involved in formation of salt bridges/ hydrogen bonds with propionic groups from phycocyanobilin molecule, while Arg-186 is more likely to form hydrogen bond with lactam ring carbonyl oxygen. Further, polar groups from Tyr-138, Glu-141, Arg-145, Tyr-161 and Asp-183 can be involved in hydrogen bonding interactions with the ligand. It is interesting to note the potential for stacking between aromatic rings of Phe-134 and Tyr-138 and pyrrole rings of the phycocyanobilin (Fig. 2C). This binding site is in the close proximity to the binding site for $4 Z, 15 E$-bilirubin-IX $\alpha$ isomer previously determined by crystallographic analysis, with marked similarity in amino acid residues binding pattern. ${ }^{15}$

It is interesting to note that FA1 binding site for myristic acid and proposed phycocyanobilin IB binding site share almost the same amino acid residues lining the cavity wall of subdomain IB (PDB code 1BJ5). ${ }^{33}$ Indeed, presence of myristic acid at FA1 binding site preclude phycocyanobilin binding to IB site, while its presence at the other FA binding sites does not influence binding of phycocyanobilin (Table S4 of ESI†). IB binding site is not large enough to accommodate ligand and myristic acid, so the ligand is pushed away towards the cleft binding site 
Table 1 Binding energies ( $\mathrm{kcal} \mathrm{mol}^{-1}$ ) for HSA-phycocyanobilin and HSA-bilirubin interactions

\begin{tabular}{llllllll}
\hline & \multicolumn{2}{l}{ Phycocyanobilin form } & & & \multicolumn{2}{l}{ Bilirubin form } \\
\cline { 2 - 3 } \cline { 5 - 7 } HSA subdomain & Neutral & Monoanionic & Dianionic & & Neutral & Monoanionic & Dianionic \\
\hline IB & 10.8 & 10.9 & 11.3 & 11.1 & 11.1 & 11.3 \\
IIA & 10.7 & 10.8 & 11.1 & 11.0 & 11.1 & 11.2
\end{tabular}

(Fig. S3A of ESI $\uparrow$ ). Binding of other ligands (such as hemin, $4 Z, 15 E$-bilirubin-IXalpha, fusidic acid) occupying IB site also prevent phycocyanobilin binding (Table S5 of ESI†े).

The second high-affinity binding site is located in the hydrophobic cavity formed by the helices H1-H5 of the IIA subdomain (Fig. 2D). This cavity is formed by the side chains of the amino acids Phe-211, Trp-214, Leu-219, Phe-223 (from H1), Val-235, Leu-238 (from H2), Leu-260, Ile-264 (from H3) and Ile290, Ala-291 (from H5). Similarly to the first binding site, this binding site is L-shaped with phycocyanobilin bound as $M$-conformer. Both highly polar/anionic propionic groups of the phycocyanobilin ligand are oriented towards upper part of the helix H10 (residues 194-206) from IB subdomain. Lys-195 and Lys-199 residues (from helix H10) are most probably positively charged. His-242 is involved in formation of salt bridges/ hydrogen bonds with propionic groups from the ligand. Other charged residues found in the binding site (Arg-218, Arg-222 and Arg-257) are involved in hydrogen bonding with carbonyl or $\mathrm{NH}$ group of lactam ring. Based on our docking analysis, there is a potential for head to tail stacking interaction between Trp-214 and one of the phycocyanobilin ligand pyrrole rings (Fig. 2D). Above-described data for phycocyanobilin docking to subdomain IIA fully correspond to proposed structure of bilirubin binding site for this subdomain on HSA molecule. ${ }^{16}$

Although phycocyanobilin position and binding energy in IIA binding site is somewhat altered by the presence of the myristic acid at the FA7 site, docking simulation predicts this binding site to be most probably binding site (Fig. S3B of ESI $\dagger$ ). The presence of myristic acid on all other HSA binding sites does not influence phycocyanobilin binding to IIA site (Table S4 of ESI $\dagger$ ), while binding of other selected ligands occupying IIA site differently influence phycocyanobilin binding. For the most of the structures, existence of ligands at IIA binding site, including R- and S-warfarin, prevents docking of phycocyanobilin. In the case of some ligands (azapropazone, azapropazone and indomethacin or lysophasphatidylethanolamine) there is still enough space to accommodate phycocyanobilin and pigment binding is not prevented (Table S5 and Fig. S4 of ESI $\dagger$ ). Especially interesting are docking results for the HSA crystal structure with two ligands (indomethacine and azapropazone; PBD code 2BXK) and phycocyanobilin docked in the same cavity (Fig. S4B of ESI†), confirming how large and versatile HSA IIA binding site is. ${ }^{8}$

Our data thus evidenced that putative binding sites on HSA for phycocyanobilin are in close proximity to binding sites experimentally demonstrated for bilirubin (Fig. S2C of ESI†). The charge state of both pigments does not influence binding mode or estimated binding energy that also suggests high affinity binding for phycocyanobilin. Furthermore, computational predictions provided a stereo-selective binding of $M$ conformer of phycocyanobilin to HSA.

Earlier approach to mutate HSA and determine critical amino acids for bilirubin binding within the domain IIA was not successful. The approach was based on predictions of dianionic form binding to HSA, thereby K195M, K199M, F211V, W214L，R218M，R222M，H242V，R257M mutations were performed within the site IIA and did not influence the binding affinity of bilirubin to HSA. ${ }^{8}$ It was proposed that the binding mode for bilirubin may be dynamic. Multiple pattern binding mode is attributable to the formation of a flexible $4 Z, 15 Z$-bilirubin-HSA complex, and no single crucial binding mode exists, but multiple interactions are ongoing between $4 Z, 15 Z$-bilirubin and HSA.

Another study employing creation of phage-displayed HSA mutants demonstrated that the binding affinity of $4 Z, 15 Z$-bilirubin for wild-type domain I (residues 1-186) was significantly lower than that for wild-type domain II. Furthermore, $4 Z, 15 Z$-bilirubin, when bound to domain I, adopts an unusual $M$-conformation that is quite different from the typical $P$-conformation that it adopts when binding to domain II and full-length HSA. ${ }^{11}$

\section{Phycocyanobilin binds to HSA and quenches protein intrinsic fluorescence}

Quenching of HSA intrinsic fluorescence, mostly contributed by Trp-214 at subdomain IIA, is useful tool for characterization of ligand binding to HSA. ${ }^{34}$ HSA has only one Trp residue and 18 Tyr residues. ${ }^{35}$ Fig. 3A shows the fluorescence emission spectra of HSA in the absence and presence of phycocyanobilin. Upon excitation of HSA at $280 \mathrm{~nm}$, protein exhibits a strong fluorescence emission with a maximum at $340 \mathrm{~nm}$ that was quenched upon phycocyanobilin addition in a concentrationdependent manner. Furthermore, a significant blue shift is observed with increasing phycocyanobilin concentration (Fig. 3A), suggesting that the intrinsic fluorophore of HSA is placed in a more hydrophobic environment after the addition of phycocyanobilin..$^{36}$ The blue shift could also be explained by a preferential quenching of the Trp-214 residues by phycocyanobilin, that would remain only Tyr residues to contribute to HSA fluorescence. Indeed, intensive quenching of Trp-214 fluorescence by phycocyanobilin makes more visible contribution of Tyr-263 to protein fluorescence pick ("shoulder" at $308 \mathrm{~nm}$; Fig. 3A). This tyrosine residue is the closest tyrosine to Sudlow 
site I located in subdomain IIA, not completely buried inside the protein. ${ }^{37}$

Fig. 3B represents the SV plots for the HSA fluorescence quenching by phycocyanobilin at three different temperatures. It can be seen that the quenching constant (slope of curves) decreases with increasing temperature (Table 2), and the values of $k_{\mathrm{q}}$ are greater than the limiting diffusion rate constant of the biomolecule $\left(2 \times 10^{10} \mathrm{M}^{-1} \mathrm{~s}^{-1}\right)$ indicating a static type of quenching. ${ }^{38}$

Phycocyanobilin binds to HSA with the binding affinities of $2.2 \times 10^{6} \mathrm{M}^{-1}$ (at $25{ }^{\circ} \mathrm{C}$ ) and approximate $1: 1$ stoichiometry (Table 2; Fig. S5 in ESI†). In comparison with drugs and other exogenous compounds, observed phycocyanobilin association constant for HSA of the order of magnitude $10^{6} \mathrm{M}^{-1}$ is among the largest, ${ }^{39}$ and comparable to that of bilirubin $\left(5 \times 10^{6} \mathrm{M}^{-1}\right.$ at $\left.25{ }^{\circ} \mathrm{C}\right) .{ }^{40}$

\section{The binding mode between HSA and phycocyanobilin}

In order to confirm binding mode of phycocyanobilin to HSA we calculated the thermodynamic parameters, enthalpy $(\Delta H)$ and entropy $(\Delta S)$ of interactions. The temperature dependence of
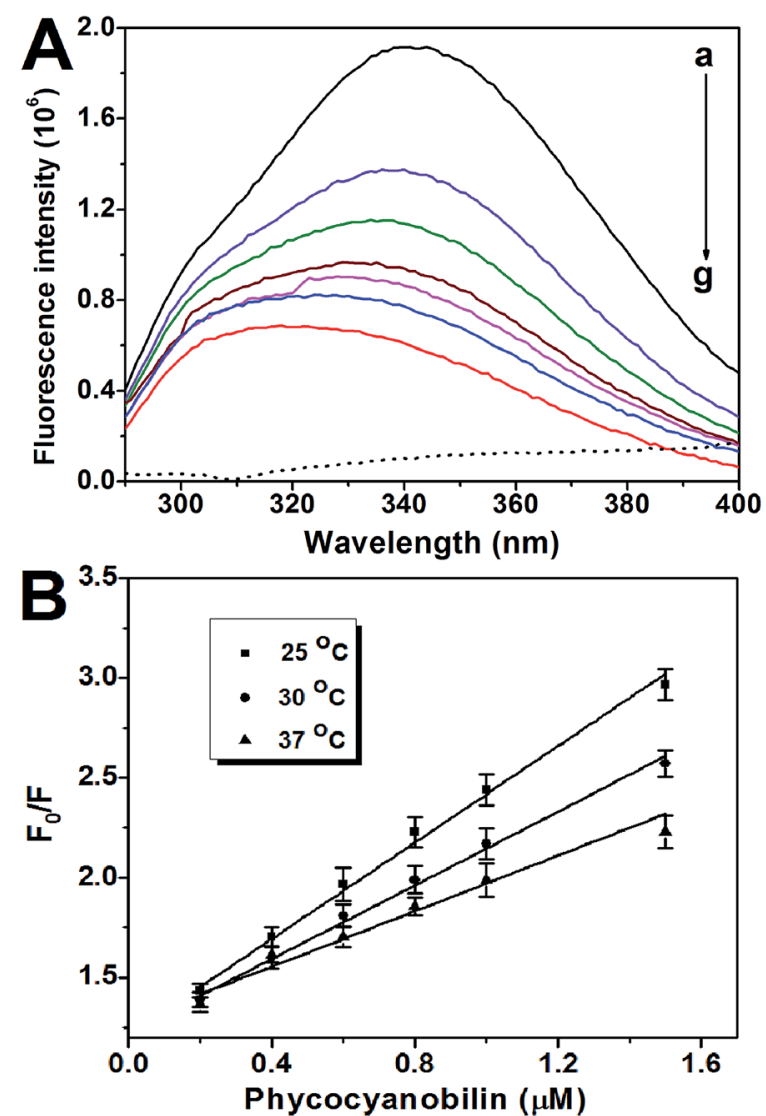

Fig. 3 (A) Emission spectra (excitation at $280 \mathrm{~nm}$ ) of HSA $(0.375 \mu \mathrm{M})$ in the presence of different concentrations of phycocyanobilin $(0,0.2$, $0.4,0.6,0.8,1.0$, and $1.5 \mu \mathrm{M}$, for curves a to $\mathrm{g}$, respectively). Dot line curve shows the emission spectrum of $1.5 \mu \mathrm{M}$ phycocyanobilin; (B) Stern-Volmer plots of HSA fluorescence quenched by phycocyanobilin at three different temperatures and $\mathrm{pH}$ 7.4. Error bars represent the standard deviation.
Table 2 The Stern-Volmer quenching constants $\left(K_{\mathrm{SV}}\right)$, bimolecular quenching constants $\left(k_{\mathrm{q}}\right)$, binding constant $\left(K_{\mathrm{a}}\right)$ and the number of binding sites $(n)$ on the HSA-phycocyanobilin system at different temperatures $^{a}$

\begin{tabular}{|c|c|c|c|c|c|c|}
\hline \multirow[b]{2}{*}{$\begin{array}{l}T \\
\left({ }^{\circ} \mathrm{C}\right)\end{array}$} & \multicolumn{3}{|l|}{ Eqn (2) } & \multicolumn{3}{|l|}{ Eqn (3) } \\
\hline & $\begin{array}{l}K_{\mathrm{SV}} \\
\left(\mathrm{M}^{-1}\right) \times 10^{6}\end{array}$ & $\begin{array}{l}k_{\mathrm{q}} \\
\left(\mathrm{M}^{-1} \mathrm{~s}^{-1}\right) \times 10^{14}\end{array}$ & S.D. & $\begin{array}{l}K_{\mathrm{a}} \\
\left(\mathrm{M}^{-1}\right) \times 10^{6}\end{array}$ & $n$ & S.D. \\
\hline 25 & 1.23 & 1.23 & 0.024 & 2.24 & 0.97 & 0.041 \\
\hline 30 & 0.92 & 0.92 & 0.018 & 1.80 & 0.83 & 0.037 \\
\hline 37 & 0.69 & 0.69 & 0.021 & 1.23 & 0.85 & 0.040 \\
\hline
\end{tabular}

${ }^{a}$ S.D. is the standard deviation.

the HSA-phycocyanobilin binding constant was studied at three different temperatures $\left(25,30\right.$ and $\left.37^{\circ} \mathrm{C}\right)$ : the plot of $\ln K_{\mathrm{a}}$ vs. $1 / T$ (Fig. S6 in ESI $\dagger$ ) enabled the determination of $\Delta H$ $\left(-39.2 \mathrm{~kJ} \mathrm{~mol}^{-1}\right)$ and $\Delta S\left(-9.7 \mathrm{~J} \mathrm{~mol}^{-1} \mathrm{~K}^{-1}\right)$.

Large negative value for $\Delta H$ and much smaller, but also negative value for $\Delta S$, at all temperatures, suggests that formation of complex is enthalpy driven and hydrogen bonding plays a significant role in the binding mode. ${ }^{41}$ Similar binding mode was previously demonstrated for HSA-bilirubin system, with a decrease in binding constant for increasing salt concentration, implicating that hydrogen bonds and electrostatic interactions are the main factor in the binding of bilirubin to its primary site on HSA. ${ }^{\mathbf{1 3}}$ Moreover, a numerous hydrogen bonds between phycocyanobilin and HSA proposed by docking analysis at both binding sites identified in our study (Fig. 2) are in agreement with experimentally determined thermodynamic data.

\section{Binding of phycocyanobilin to HSA changes environment of Trp-214 residue}

Synchronous fluorescence spectroscopy gives information about the molecular environment in the vicinity of the fluorophore functional groups. When $\Delta \lambda$ between the excitation wavelength and the emission wavelength were set at 15 or $60 \mathrm{~nm}$, the synchronous fluorescence could provide information of the tyrosine or tryptophan residues environment in HSA, respectively. ${ }^{42}$ In contrast to Tyr residues (Fig. $4 \mathrm{~A}$ ), a small blue shift (from 343 to $340 \mathrm{~nm}$ ) in the maximum emission wavelength of Trp residues was observed (Fig. 4B). This indicates the polarity around the Trp residue is decreased, while the microenvironment around the tyrosine residues did not significantly change during the binding process. It has also been shown in Fig. 4C that the slope of SV quenching plot was sharper when $\Delta \lambda$ was $60 \mathrm{~nm}$, confirming that protein fluorescence decrease upon phycocyanobilin binding mostly originates from the quenching of critical Trp-214 residue. $^{43}$

Phycocyanobilin efficiently quenched Trp and placed it in a more hydrophobic environment (as demonstrated in quenching experiments, as well as in synchronous spectra), and this effect arise from $\pi$-stacking interaction between Trp-214 and phycocyanobilin. 

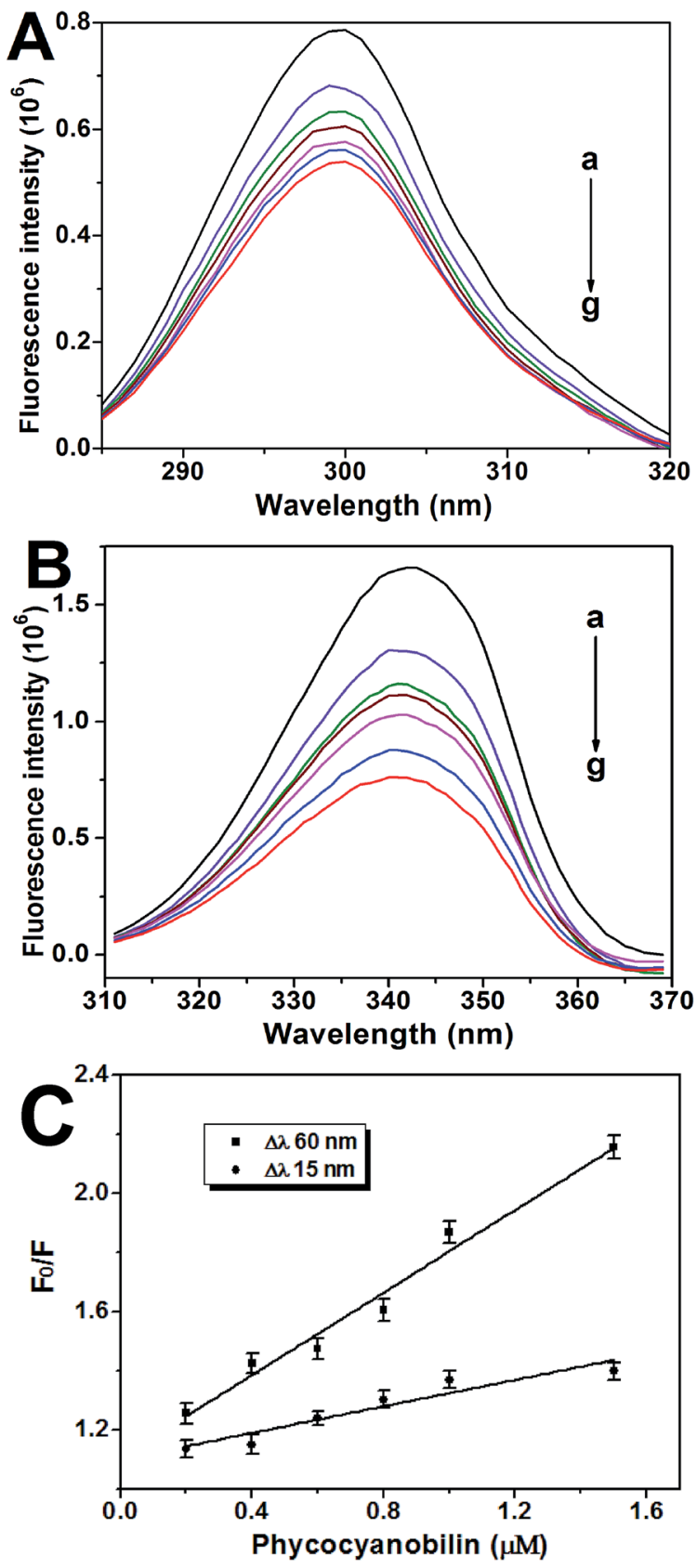

Fig. 4 Synchronous fluorescence spectra of HSA $(0.375 \mu \mathrm{M})$ with (A) $\Delta \lambda=15 \mathrm{~nm}$ (Tyr) and with (B) $\Delta \lambda=60 \mathrm{~nm}$ (Trp) in the presence of increasing concentration of phycocyanobilin $(0-1.5 \mu \mathrm{M})$ for curves a to g, respectively; (C) the Stern-Volmer quenching plots of HSA synchronous fluorescence by phycocyanobilin: $\Delta \lambda=60 \mathrm{~nm}$ for Trp and $\Delta \lambda=15 \mathrm{~nm}$ for Tyr. Error bars represent standard deviation.

Binding of phycocyanobilin to HSA induces enhancement of pigment fluorescence and negative Cotton effect in CD spectrum of phycocyanobilin

Phycocyanobilin is a chromophore that shows characteristic UV-visible, CD and fluorescence spectra. It is known that upon interaction of its analog bilirubin with serum albumin, there is a significant enhancement of weak intrinsic fluorescence and a red shift of the emission maximum..$^{44,45}$
Fig. 5A represents the fluorescence spectra of phycocyanobilin after excitation at $460 \mathrm{~nm}$. Upon HSA addition, strong fluorescence of phycocyanobilin further increases with the maximum emission shift towards higher wavelengths (from 518 to $541 \mathrm{~nm}$ ). Calculated binding constant from the enhancement of fluorescence was $1.1 \times 10^{5} \mathrm{M}^{-1}$ (Fig. S7 $\dagger$ ).

Phycocyanobilin exists mostly in ZZZsss (all $Z$, all syn) conformation, adopting a cyclic-helical conformation with a right-hand $(P)$ and left-hand $(M)$ conformers in equilibrium. ${ }^{46}$ Therefore, at $\mathrm{pH} 7.4$ phycocyanobilin does not show optical activity. HSA addition generates a positive band at $370 \mathrm{~nm}$ and a negative band at $620 \mathrm{~nm}$ in the $\mathrm{CD}$ spectrum of phycocyanobilin, producing so-called negative Cotton effect (Fig. 5B). This result indicates that shifting of equilibrium between $P$ and $M$ conformers, as a consequence of binding of one of them for a protein, causes optical activity of phycocyanobilin. Similarly to our results on phycocyanobilin, the consequence of bilirubin and biliverdin $M$ conformers binding for HSA produces a negative Cotton effect. ${ }^{10,11,47}$ The right-handed, $P$-helical conformer of biliverdin displays a negative Cotton effect near $380 \mathrm{~nm}$ and a positive Cotton effect around $650 \mathrm{~nm} .{ }^{48}$ Therefore, our result proves binding of phycocyanobilin $M$-conformer to
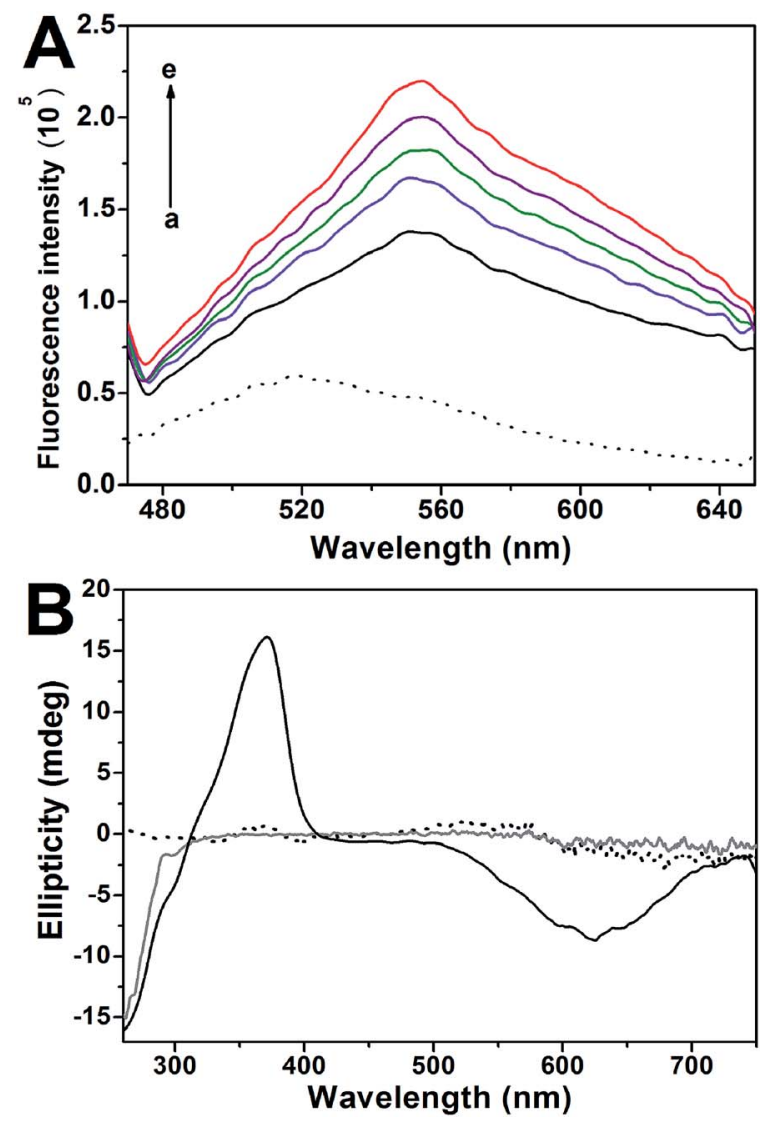

Fig. 5 (A) Effect of HSA addition (1, 1.25, 1.5, 1.75, and $2 \mu \mathrm{M}$, for curves a to e, respectively) on the emission spectra of $1 \mu \mathrm{M}$ phycocyanobilin (excitation at $460 \mathrm{~nm}$ ). Dot line curve: the emission spectrum of $1 \mu \mathrm{M}$ phycocyanobilin; (B) near UV-visible CD spectra of phycocyanobilin $(18 \mu \mathrm{M})$ in the presence (black solid line) and absence of $18 \mu \mathrm{M}$ HSA (dot line). Gray line represents CD spectrum of $18 \mu \mathrm{M} \mathrm{HSA}$. 
the protein and these results are in line with the computational predictions.

Analysis of the CD spectral changes during $\mathrm{NaBH}_{4}$ reduction of biliverdin bound noncovalently to HSA indicates an in situ inversion of the predominantly $M$-helicity verdin conformation to afford a predominantly $P$-chirality bilirubin bound to the protein. ${ }^{\mathbf{1 0}}$ It is thus proposed that the binding site for bilirubin/ biliverdin may be flexible enough to support transition of $M$-helical verdin conformer to left-helical $P$-enantiomer of bilirubin.

\section{Phycocyanobilin competes with bilirubin, and site markers warfarin and hemin for binding to HSA}

Taking into account the structural similarity, the results of docking studies, and experimentally determined binding affinity of phycocyanobilin to HSA, an additional study was performed to confirm identity of binding site(s) for phycocyanobilin and bilirubin on HSA molecule.

In these experiments, we firstly monitored various spectral changes of HSA-bilirubin complex after phycocyanobilin addition and vice versa. Excitation of HSA-bilirubin and HSA-phycocyanobilin complexes at $460 \mathrm{~nm}$ induces strong emission maxima at relatively close wavelength at 516 and $547 \mathrm{~nm}$, respectively. Therefore, synchronous fluorescence spectra were obtained with $\Delta \lambda 56 \mathrm{~nm}$ and $\Delta \lambda 87 \mathrm{~nm}$, for monitoring emission of HSA-bilirubin and HSA-phycocyanobilin complexes, respectively. When $\Delta \lambda$ is set at $56 \mathrm{~nm}$, bilirubin in the complex with HSA shows strong emission peak at $523 \mathrm{~nm}$. Free bilirubin at these conditions has negligible emission (Fig. 6A). Upon phycocyanobilin addition, peak intensity markedly decreases suggesting increases of free bilirubin concentration due to phycocyanobilin displacement of bilirubin from its binding site on HSA. This result also supports binding constants similarity of bilirubin and phycocyanobilin for HSA. When $\Delta \lambda$ is set at $87 \mathrm{~nm}$, emission peak of HSA-phycocyanobilin complex appears at $618 \mathrm{~nm}$. At this wavelength, HSA-phycocyanobilin complex has notably larger emission in comparison to free phycocyanobilin (Fig. 6B). Upon bilirubin addition, peak intensity decreases, indicating that free phycocyanobilin concentration increases as bilirubin induces displacement of phycocyanobilin from its binding site on HSA.

Bilirubin in the complex with HSA shows optical activity, with the positive and negative band in near UV-visible CD spectra at 460 and $410 \mathrm{~nm}$, respectively. ${ }^{16}$ From Fig. 6C it can be seen that the addition of bilirubin in a solution containing HSA-phycocyanobilin complex leads to a reduction of the ellipticity at $370 \mathrm{~nm}$, as well as the decrease in ellipticity at 620 $\mathrm{nm}$. Knowing that both bands originate from phycocyanobilin bound to HSA and phycocyanobilin itself does not show optical activity (Fig. 6C) these data additionally support phycocyanobilin displacement by bilirubin from its complex with HSA.

The ligands (probes) that specifically bind to a known region on HSA are often used in competition experiments with the tested substance to facilitate the identification of the binding site. X-ray crystallography studies unequivocally uncovered that warfarin is probe for subdomain IIA $^{49}$ and hemin is probe for subdomain IB. ${ }^{50}$ Upon warfarin addition to HSA-phycocyanobilin in 1:1 molar ratio, small decrease of emission maximum appears, wherein at tenfold excess warfarin induces only slight additional decrease of peak intensity (Fig. 6D). In contrast, phycocyanobilin efficiently displace warfarin from HSA-warfarin equimolar complex (Fig. 6E). Under the same experimental design, similar results can be observed in the case of warfarin competition with bilirubin for HSA and vice versa (Fig. S8 and S9 of $\mathrm{ESI}^{\dagger}$ ). The results may be explained by the fact that warfarin has much smaller binding constant for HSA $\left(3.8 \times 10^{5} \mathrm{M}^{-1}\right.$ at $\left.25^{\circ}\right)$ in comparison to bilirubin $\left(5.0 \times 10^{6} \mathrm{M}^{-1}\right.$ at $\left.25^{\circ}\right)$ and phycocyanobilin $\left(2.2 \times 10^{6} \mathrm{M}^{-1}\right.$; Table 2$)$. Re-docking simulation on 1HA2 and 2BXD structures resulted in $R$ - and $S$-warfarin binding energies of 9.6 and $8.8 \mathrm{kcal} \mathrm{mol}^{-1}$, respectively, $1.2-2.0 \mathrm{kcal}$ $\mathrm{mol}^{-1}$ lower values (Table 1) then phycocyanobilin binding energy in IIA site. Titration of equimolar HSA-phycocyanobilin mixture with hemin (binding constant of $0.9 \times 10^{6} \mathrm{M}^{-1}$ at $25^{\circ}$ ) causes powerful fluorescence signal quenching of the pigment in a complex with a protein (Fig. 6F). In fact, the same observation can be seen after bolus addition of hemin into equimolar mixture of HSA with bilirubin (Fig. S10 $\dagger$ ). This suggests that these ligands bind not only to subdomain IIA in the Sudlow site 1 but also to subdomain IB, the respective binding site of warfarin and hemin.

In order to compare the effect of site markers on HSA-phycocyanobilin binding, phycocyanobilin binding constants in the presence of site markers were calculated using eqn (3), and compared to the original value. The phycocyanobilin binding constant $\left(2.2 \times 10^{6} \mathrm{M}^{-1}\right)$ was only slightly decreased in the presence of warfarin $\left(1.6 \times 10^{6} \mathrm{M}^{-1}\right)$ or hemin $\left(2.0 \times 10^{6} \mathrm{M}^{-1}\right)$. However, in the presence of both markers binding constant was notably reduced $\left(0.9 \times 10^{5} \mathrm{M}^{-1}\right)$. These results suggests that in the presence of warfarin phycocyanobilin can bind freely to IB subdomain binding site, but to compete with warfarin for IIA binding site resulting in apparently slightly reduced binding constant. Similarly, the presence of hemin in system leaves free binding site IIA resulting also in slightly decreased phycocyanobilin binding constant for HSA. When both of the markers are present phycocyanobilin competes with them for both available high-affinity binding sites on protein, leading to significantly reduced binding constant. In addition, although warfarin has one order of magnitude lower $K_{\mathrm{a}}$ then hemin (see previous paragraph), it is more efficient in reducing phycocyanobilin binding constant for HSA in comparison to hemin, indicating that phycocyanobilin affinity to binding site IIA is somewhat higher than for binding site IB.

It has previously been shown that bile pigment structurally similar to phycocyanobilin, biliverdin, also competes with bilirubin, but only for the high affinity binding site. ${ }^{48}$ Previous data have shown that an addition of a competitor ligand (drug specific for high affinity site) to bilirubin-HSA complex caused a redistribution of the bilirubin among the other sites which lead to a less efficient competition for binding to the protein. ${ }^{\mathbf{1 6}}$ Taken together, our results of displacement experiments further advocated identity of binding sites for phycocyanobilin and bilirubin on HSA, and propensity of phycocyanobilin to efficiently compete for the binding to the major plasma carrier protein. 

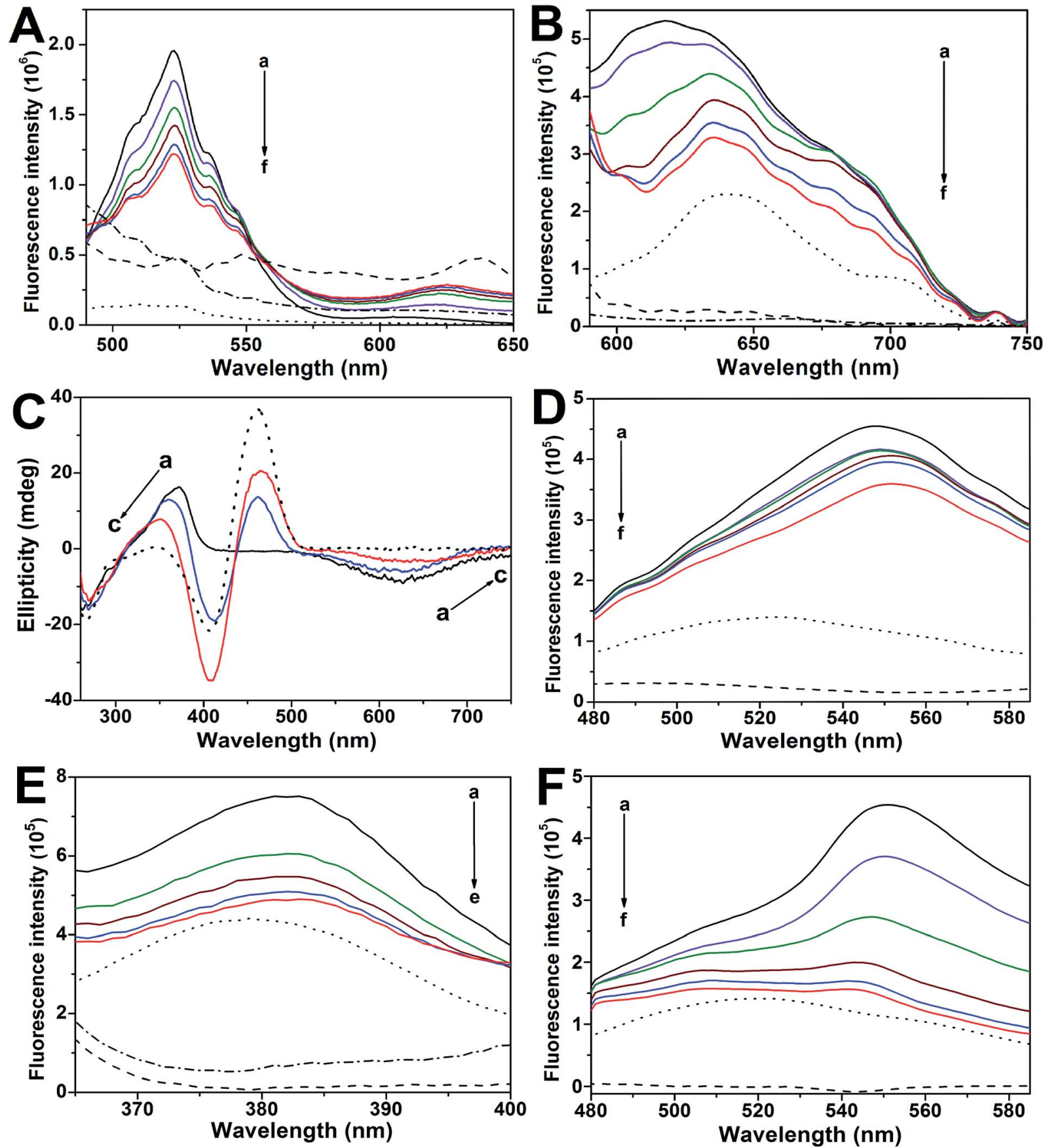

Fig. 6 (A) Quenching of HSA-bilirubin complex ( $4 \mu \mathrm{M}$ both) by phycocyanobilin $(0,2,4,6,8$ and $10 \mu \mathrm{M}$, for curves a to f, respectively) using synchronous fluorescence spectroscopy, $\Delta \lambda 56 \mathrm{~nm}$. Dash line curve: $4 \mu \mathrm{M}$ HSA in presence of $4 \mu \mathrm{M}$ phycocyanobilin. Dot line curve: $10 \mu \mathrm{M}$ bilirubin. Dash-dot line curve: $10 \mu \mathrm{M}$ phycocyanobilin; (B) quenching of HSA-phycocyanobilin complex $(2 \mu \mathrm{M}$ both) by bilirubin ( $0,1,2,3,4$ and $5 \mu \mathrm{M}$, for curves a to f, respectively) using synchronous fluorescence spectroscopy, $\Delta \lambda 87 \mathrm{~nm}$. Dash line curve represents $2 \mu \mathrm{M} H S A$ in presence of $2 \mu \mathrm{M}$ bilirubin. Dot line curve: $2 \mu \mathrm{M}$ phycocyanobilin. Dash-dot line curve represents $10 \mu \mathrm{M}$ bilirubin; (C) decreasing of HSA-phycocyanobilin complex (18 $\mu \mathrm{M}$ both) ellipticity by bilirubin $(0,18$ and $36 \mu \mathrm{M}$ for curves a to $\mathrm{C}$, respectively). Dot line curve shows the CD spectrum of $18 \mu \mathrm{M}$ bilirubin in presence of $18 \mu \mathrm{M}$ HSA. (D) Quenching of HSA-phycocyanobilin complex ( $2 \mu \mathrm{M}$ both) by warfarin $(0,2,4,6,8$ and $20 \mu \mathrm{M}$, for curves a to $\mathrm{f}$, respectively) (excitation wavelength $460 \mathrm{~nm}$ ). Dash line curve: $2 \mu \mathrm{M}$ HSA in presence of $20 \mu \mathrm{M}$ warfarin. Dot line curve: $2 \mu \mathrm{M}$ phycocyanobilin; (E) quenching of HSA-warfarin complex $(0.25 \mu \mathrm{M}$ both) by phycocyanobilin $(0,0.125,0.25,0.375$, and 0.5 , for curves a to e, respectively) using synchronous fluorescence spectroscopy, $\Delta \lambda 64 \mathrm{~nm}$. Dash line curve: $0.25 \mu \mathrm{M}$ HSA. Dot line curve: $0.25 \mu \mathrm{M}$ warfarin. Dash-dot line curve: $0.25 \mu \mathrm{M} \mathrm{HSA}$ in presence of $0.25 \mu \mathrm{M}$ phycocyanobilin; (F) quenching of HSA-phycocyanobilin complex $(2 \mu \mathrm{M}$ both) by hemin $(0,1,2,3,4$, and $5 \mu \mathrm{M}$, for curves a to f, respectively) (excitation wavelength $460 \mathrm{~nm}$ ). Dot line curve: $2 \mu \mathrm{M}$ phycocyanobilin, dash line curve: $2 \mu \mathrm{M}$ HSA in presence of $2 \mu \mathrm{M}$ hemin. 


\section{Conclusions}

Our work, based on a computational approach to studying interactions between HSA, bilirubin and bilirubin-analog phycocyanobilin, demonstrated two putative high-affinity binding pockets on HSA for bilirubin and phycocyanobilin, of virtually identical energies for the neutral, mono- and dianionic forms of both molecules.

The binding sites were identical to the two previously identified binding sites for bilirubin (at subdomain IB and subdomain IIA). Results obtained by protein and pigment fluorescence measurements, circular dichroism, as well as bilirubin-displacement experiments, confirmed high-affinity, enantioselective binding of phycocyanobilin $M$-conformer to HSA and its competition with bilirubin, warfarin and hemin for the binding on HSA. Ionizing state of ligands (neutral vs. monoanion $v s$. dianion) appeared to have no marked impact on binding to HSA. The present results provide valuable information for the transportation and distribution of phycocyanobilin in vivo, which may be of importance for the understanding of its proposed numerous beneficial effects. In addition, our data suggest that in conditions characterized by an increased bilirubin plasma concentration, or intake of drugs binding to IB or IIA HSA binding site, pharmacokinetics of phycocyanobilin may also be changed.

\section{Conflict of interest}

The authors declare no conflict of interest.

\section{Acknowledgements}

This work was supported by the Ministry of Education, Science and Technological Development of the Republic of Serbia (Grant Numbers 172035 and 172024) and FP7 RegPot project FCUB ERA GA No. 256716. The EC does not share responsibility for the content of the article. We are grateful to Ms Maja Krstic for help with CD data collection.

\section{References}

1 B. Fernández-Rojas, J. Hernández-Juárez and J. PedrazaChaverri, J. Funct. Foods, 2014, 11, 375-392.

2 J. Marin-Prida, N. Pavon-Fuentes, A. Llopiz-Arzuaga, J. R. Fernandez-Masso, L. Delgado-Roche, Y. MendozaMari, S. P. Santana, A. Cruz-Ramirez, C. Valenzuela-Silva, M. Nazabal-Galvez, A. Cintado-Benitez, G. L. Pardo-Andreu, N. Polentarutti, F. Riva, E. Penton-Arias and G. Penton-Rol, Toxicol. Appl. Pharmacol., 2013, 272, 49-60.

3 R. Konickova, K. Vankova, J. Vanikova, K. Vanova, L. Muchova, I. Subhanova, M. Zadinova, J. Zelenka, A. Dvorak, M. Kolar, H. Strnad, S. Rimpelova, T. Ruml, R. J. Wong and L. Vitek, Ann. Hepatol., 2014, 13, 273-283.

4 G. Sudlow, D. J. Birkett and D. N. Wade, Mol. Pharmacol., 1975, 11, 824-832.

5 F. Zsila, Mol. Pharmacol., 2013, 10, 1668-1682.
6 T. Wybranowski, M. Cyrankiewicz, B. Ziomkowska and S. Kruszewski, BioSystems, 2008, 94, 258-262.

7 R. Brodersen, J. Biol. Chem., 1979, 254, 2364-2369.

8 C. E. Petersen, C. E. Ha, K. Harohalli, J. B. Feix and N. V. Bhagavan, J. Biol. Chem., 2000, 275, 20985-20995.

9 D. A. Lightner, M. Reisinger and G. L. Landen, J. Biol. Chem., 1986, 261, 6034-6038.

10 F. R. Trull, O. Ibars and D. A. Lightner, Arch. Biochem. Biophys., 1992, 298, 710-714.

11 A. Minomo, Y. Ishima, U. Kragh-Hansen, V. T. Chuang, M. Uchida, K. Taguchi, H. Watanabe, T. Maruyama, H. Morioka and M. Otagiri, FEBS J., 2011, 278, 4100-4111.

12 A. Kumagai, R. Ando, H. Miyatake, P. Greimel, T. Kobayashi, Y. Hirabayashi, T. Shimogori and A. Miyawaki, Cell, 2013, 153, 1602-1611.

13 J. Jacobsen, Int. J. Pept. Protein Res., 1977, 9, 235-239.

14 M. J. Maisels and A. F. McDonagh, N. Engl. J. Med., 2008, 358, 920-928.

15 P. A. Zunszain, J. Ghuman, A. F. McDonagh and S. Curry, J. Mol. Biol., 2008, 381, 394-406.

16 I. Goncharova, S. Orlov and M. Urbanova, Biophys. Chem., 2013, 180-181, 55-65.

17 P. A. Zunszain, J. Ghuman, T. Komatsu, E. Tsuchida and S. Curry, BMC Struct. Biol., 2003, 3, 6.

18 M. Dockal, D. C. Carter and F. Ruker, J. Biol. Chem., 1999, 274, 29303-29310.

19 P. Mukerjee and J. D. Ostrow, BMC Biochem., 2010, 11, 16.

20 S. E. Boiadjiev and D. A. Lightner, Tetrahedron: Asymmetry, 1999, 10, 607-655.

21 E. Fu, L. Friedman and H. W. Siegelman, Biochem. J., 1979, 179, 1-6.

22 W. J. Cole, D. J. Chapman and H. W. Siegelman, J. Am. Chem. Soc., 1967, 89(14), 3643-3645.

23 S. Sastre, R. Casasnovas, F. Muñoz and J. Frau, Theor. Chem. Acc., 2012, 132, 1-8.

24 A. V. Marenich, C. J. Cramer and D. G. Truhlar, J. Phys. Chem. B, 2009, 113, 6378-6396.

25 S. Sugio, A. Kashima, S. Mochizuki, M. Noda and K. Kobayashi, Protein Eng., 1999, 12, 439-446.

26 R. Anandakrishnan, B. Aguilar and A. V. Onufriev, Nucleic Acids Res., 2012, 40, W537-W541.

27 B. R. Brooks, R. E. Bruccoleri, B. D. Olafson, D. J. States, S. Swaminathan and M. Karplus, J. Comput. Chem., 1983, 4, 187-217.

28 O. Trott and A. J. Olson, J. Comput. Chem., 2010, 31, 455-461. 29 J. Ghuman, P. A. Zunszain, I. Petitpas, A. A. Bhattacharya, M. Otagiri and S. Curry, J. Mol. Biol., 2005, 353, 38-52.

$30 \mathrm{~J}$. R. Lakowicz, Principles of Fluorescence Spectroscopy, Springer, New York, 2006.

31 S. Bia, L. Dinga, Y. Tiana, D. Songa, X. Zhoua, X. Liua and H. Zhanga, J. Mol. Struct., 2004, 703, 37-45.

32 L. Liang, H. A. Tajmir-Riahi and M. Subirade, Biomacromolecules, 2008, 9, 50-56.

33 S. Curry, H. Mandelkow, P. Crick and N. Franks, Nat. Struct. Biol., 1998, 5, 827-835.

34 A. Sułkowska, J. Mol. Struct., 2002, 614, 227-232.

35 X. M. He and D. C. Carter, Nature, 1992, 358, 209-215. 
36 Z. Chi and R. Liu, Biomacromolecules, 2011, 12, 203-209.

37 O. K. Abou-Zied and O. I. K. Al-Shihi, J. Am. Chem. Soc., 2008, 130, 10793-10801.

38 X. Li and S. Wang, New J. Chem., 2015, 39, 386-395.

39 A. Varshney, P. Sen, E. Ahmad, M. Rehan, N. Subbarao and R. H. Khan, Chirality, 2010, 22, 77-87.

40 A. A. Lamola, J. Eisinger, W. E. Blumberg, S. C. Patel and J. Flores, Anal. Biochem., 1979, 100, 25-42.

41 P. D. Ross and S. Subramanian, Biochemistry, 1981, 20, 30963102.

42 J. N. Miller, Proc. Anal. Div. Chem. Soc., 1979, 16, 203-208.

43 J. Zhang, S. Zhuang, C. Tong and W. Liu, J. Agric. Food Chem., 2013, 61, 7203-7211.
44 M. M. Khan, S. Muzammil and S. Tayyab, Biochim. Biophys. Acta, 2000, 1479, 103-113.

45 Z. Hrkal and S. Klementova, Int. J. Biochem., 1984, 16, 799804.

46 P.-h. Tu, Y.-h. Yao, Y.-l. Li and B. Liu, J. Mol. Struct.: THEOCHEM, 2009, 894, 9-13.

47 Y.-M. Pu, A. F. McDonagh and D. Lightner, J. Am. Chem. Soc., 1993, 15, 377-380.

48 C. E. Ahlfors, Anal. Biochem., 1981, 110, 295-307.

49 I. Petitpas, A. A. Bhattacharya, S. Twine, M. East and S. Curry, J. Biol. Chem., 2001, 276, 22804-22809.

50 M. Dockal, D. C. Carter and F. Rüker, J. Biol. Chem., 1999, 274, 29303-29310. 